DOI: $10.5965 / 25946412222018135$

\title{
SENSIBILIZANDO ADOLESCENTES PARA A RESPONSABILIDADE SOCIAL DA MATERNIDADE-PATERNIDADE PRECOCE
}

Roberta dos Santos Ribeiro

Universidade Estadual de Santa Cruz robertaribeiro31@gmail.com

Simone Setúbal dos Santos Universidade Estadual de Santa Cruz monvset@hotmail.com

Danielle Oliveira dos Anjos Universidade Estadual de Santa Cruz dannvanjos@gmail.com

Andréa Gonçalves dos Santos Universidade Estadual de Santa Cruz andreadiscipula@hotmail.com

Rutilea Mendes de Morais Universidade Estadual de Santa Cruz rutidiscipula@gmail.com

Jamilly Azevedo Sena

Universidade Estadual de Santa Cruz millvbio7@gmail.com

Silvia Maria Santos Carvalho Universidade Estadual de Santa Cruz sissa@uesc.br

Jane Lima dos Santos Universidade Estadual de Santa Cruz ilsantos@uesc.br

Aparecida Zerbo Universidade Estadual de Santa Cruz zerbotremacoldi@uesc.br

\section{Resumo}

Embora as informações sobre sexualidade e contracepção pareçam estar bastante disponíveis entre os 
adolescentes, a gravidez precoce ainda é um problema de saúde pública e de grandes impactos sociais. Nesse sentido, a escola desempenha um papel fundamental, não apenas na compreensão de processos biológicos, como também na conscientização do sujeito sobre os aspectos de cidadania e construção de uma sociedade sustentável. No seguinte relato apresentamos nossa experiência acerca projeto de extensão intitulado "despertando a consciência para o impacto individual e social da maternidade/paternidade na adolescência", desenvolvido em uma escola pública do município de IlhéusBA. Visamos realizar atividades de conscientização da gravidez precoce, em três encontros, dispondo da utilização de métodos lúdicos, oficina, debates e avaliação quantitativa e qualitativa sobre o tema, bem como sensibilizar para o desenvolvimento de outras atividades importantes a serem focadas no processo da infância/adolescência.

Palavras-chave: Gravidez precoce. Educação em saúde. Cidadania.

\title{
SENSITIZING ADOLESCENTS FOR THE SOCIAL RESPONSIBILITY OF EARLY PATERNITY-MATERNITY
}

\begin{abstract}
Teenage pregnancy remains a serious social and economic problem in brazil. In this perspective, the school plays an important role in the education of individuals not only in biological aspect but to in education for one healthy sexuality in economic and social aspect. In our work, presenting our experience about the extension project "raising awareness for the individual and social impact of maternity/paternity in adolescence" developed in one municipal public school in the municipally of Ilheus, Bahia, Brazil. We used interactive plays, workshops, debate and quantitative and qualitative assessment with proposing to make sensitized about early maternity/paternity and present new life perspectives to develop in childhood and adolescence.
\end{abstract}

Keywords: teenage pregnancy. Health education. Citizenship.

\section{SENSIBILIZANDO ADOLESCENTES PARA LA RESPONSABILIDAD SOCIAL DE LA MATERNIDAD-PATERNIDAD PRECOCE}

\section{Resumem}

Aunque la información sobre sexualidad y anticoncepción parece estar bastante disponible entre los adolescentes, el embarazo precoz sigue siendo un problema de salud pública y de grandes impactos sociales. En este sentido, la escuela desempeña un papel fundamental, no sólo en la comprensión de procesos biológicos, sino también en la concientización del sujeto sobre los aspectos de ciudadanía y construcción de una sociedad sostenible. En el siguiente relato presentamos nuestra experiencia sobre proyecto de extensión titulado "despertando la conciencia para el impacto individual y social de la maternidad/paternidad en la adolescencia", desarrollado en una escuela pública del municipio de islotes. En los tres encuentros, con el uso de métodos lúdicos, taller, debates y evaluación cuantitativa y cualitativa sobre el tema, así como sensibilizar para el desarrollo de otras actividades importantes a ser enfocadas en el proceso de la infancia / adolescencia.

Palabras clave: Embarazo precoz. Educación en salud. Ciudadanía. 


\section{INTRODUÇÃO}

O presente relato de experiência é parte das atividades desenvolvidas no projeto de extensão intitulado "Despertando a consciência para o impacto individual e social da maternidade/paternidade na adolescência", desenvolvido por docentes e discentes da Universidade Estadual de Santa Cruz, Ilhéus-BA. A proposta do projeto foi motivada pela observação na cidade de Ilhéus e região do número de adolescentes grávidas ou mães muito jovens com um ou mais filhos e pelas informações obtidas em conversa com professoras do ensino básico, além das encontradas na literatura (Ribeiro, 2015, IPERBA, 2016) em que, normalmente, estas adolescentes/jovens, deixam de ir à escola.

Embora a taxa de fecundidade no Brasil tem decrescido ao longo dos anos (entre 2004 2014 a queda foi de 18,6\%), com a Política Nacional de Planejamento Familiar, oferecendo contraceptivos gratuitos ou por aquisição a baixo custo em Farmácia Popular, o Ministério da Saúde (MS) relatou que a taxa de fecundidade entre adolescentes, aumentou $26 \%$ desde a década de 1990 e que houve um aumento nos índices de atendimento do SUS às internações para atendimento obstétrico nas faixas etárias de 10 a 14, 15 a 19 e 20 a 24 anos, com $37 \%$ das internações por gravidez, parto e puerpério entre mulheres de 10 a 19 anos (Silva et al., 2010). Por outro lado, Rebouças (2014) relata que há casos de adolescentes que engravidam por sentirem desejo de ser mãe e constituírem família.

Segundo Ribeiro (2015), IPERBA (2016), entre as adolescentes gestantes, a maioria tem renda familiar baixa, escolaridade incompleta e interrompem os estudos durante a gravidez ou após o nascimento da criança. Ainda, de acordo com estudos das Organizações Unidas no Brasil (2017), no período entre 2015 e 2016, das crianças nascidas no País, $20 \%$ eram filhos de adolescentes, $70 \%$ eram afrodescendentes e $50 \%$ moravam na região nordeste.

Apesar de o estudo do corpo humano estar previsto no PCN do ensino fundamental II e médio, inclusive com o tema reprodução humana, Altmann (2001) alerta que a maioria das escolas não trabalha este tema e, quando aborda, o sentido biológico é priorizado, não levando em conta o aspecto holístico e social em torno da sexualidade humana.

Embora as informações sobre sexualidade e contracepção pareçam estar bastante disponíveis entre os adolescentes, segundo Carvacho, Pinto e Silva, Mello (2008) e Rocha (2009), para uma parcela desta população estas informações parecem não estarem sendo suficientes ou adequadas. Carvacho et al (2008) verificou que os adolescentes não possuíam conhecimentos suficientes a respeito da anatomia e funcionamento dos órgãos sexuais e sobre a fisiologia reprodutiva e Romero et al. (2007) destaca que a mídia (revistas, televisão e meios

Cidadania em Ação: Revista de Extensão e Cultura, Florianópolis (SC), v. 2, n.2, jul./dez. 2018. 
eletrônicos), amigos, parentes próximos e os pais são as principais fontes de informação, o que pode gerar conhecimentos distorcidos e insuficientes.

Assim, é fundamental que a escola desenvolva atividades buscando despertar a consciência para consequências de uma gravidez não planejada para a vida dos próprios, um papel fundamental ao promover o conhecimento, explorando a vivência, bem como o interesse e a curiosidade dos alunos (BNCC, 2017). Neste sentido, a escola é o ambiente propício para uma discussão fundamentada em dados científicos para ampliar e aprofundar a discussão entre os adolescentes e jovens sobre reprodução humana em um olhar além da biologia, para que assim os jovens possam, conscientemente, fazer suas escolhas em constituir família, ou não, garantindo qualidade de vida para si e seus dependentes, bem como para a sociedade.

O presente trabalho foi desenvolvido na Escola Municipal São Pedro, Ilhéus, BA onde a grande maioria do alunado reside na zona rural, tendo muitos adolescentes vivendo em condição social desfavorável, carentes de informações adequadas, devido às possibilidades educacionais restritas, e consequentemente, sem muitas expectativas. Nesse sentido, nosso objetivo foi propor a ampliação do olhar de crianças/adolescentes sobre esta temática, procurando mostrar que há outros horizontes a serem contemplados e percorridos antes de se tornarem pais. Todavia, se optarem por constituírem família, precocemente, que possam fazer suas escolhas de forma consciente para garantia do bem-estar do indivíduo e de uma sociedade sustentável.

\section{METODOLOGIA}

As atividades realizadas com alunos do $6^{\circ}, 7^{\circ}$ e $8^{\circ}$ ano da Escola Municipal São Pedro, localizada no Bairro Salobrinho, Ilhéus-BA, foram desenvolvidas em três encontros, juntamente com a professora das turmas, com um total de 56 alunos. No primeiro encontro explicamos a proposta do projeto, convidando-os a participarem. Todos os alunos mostraramse interessados, curiosos. Iniciamos as ações aplicando o jogo interativo, com questões de conteúdo biológico, sobre o desenvolvimento embrionário, do $1^{\circ}$ ao $9^{\circ}$ mês de gestação, e sobre a saúde da mulher gestante. Apresentamos também modelos didáticos sobre fecundação, desenvolvimento embrionário e fetal. Ao fim do jogo, os alunos foram incentivados a anotarem suas dúvidas em um papel para serem discutidas no próximo encontro.

No segundo encontro, realizamos uma oficina onde as turmas foram organizadas em grupos de 4 a 5 alunos. Na primeira etapa, foi entregue a cada grupo um texto, resumido, elaborado a partir das dúvidas levantadas no encontro anterior, para que lessem e Cidadania em Ação: Revista de Extensão e Cultura, Florianópolis (SC), v. 2, n.2, jul./dez. 2018. 
confeccionassem um painel com imagens disponibilizadas, sobre fecundação, desenvolvimento embrionário e gemelaridade. Após apresentação oral dos grupos, realizamos a segunda etapa, focada na discussão ética- social da gravidez precoce. Cada grupo foi incentivado para que indicassem um representante e cada representante retirou de um envelope, aleatoriamente, uma pergunta de caráter de cidadania, sobre responsabilidade contraceptiva, machismo e fatores relacionados às responsabilidades dos pais na estrutura familiar, como com os cuidados físicos, sociais-emocionais e recursos financeiros para o desenvolvimento do indivíduo. Como elementos de apoio a formulação das respostas, foram disponibilizadas imagens de hospital, alimentos, casa, escola, lazer, interação familiar em condições para uma família estruturada e desestruturada.

$\mathrm{Na}$ tentativa de ampliar os horizontes em relação às diversas possibilidades que o adolescente/jovem tem disponível, no último encontro, os estudantes foram reunidos na Universidade Estadual de Santa Cruz - UESC, localizada a 600 metros de distância da Escola Municipal São Pedro para discussão e finalização das atividades. Para uma avaliação quantitativa, foi solicitado que respondessem um questionário com questões objetivas, de conteúdo biológico, e questões dissertativas relativas às ações educacionais e cidadania. Depois, para uma avaliação qualitativa, foi realizada uma atividade integrativa, com a passagem de um balão, ao som da música "Debaixo d'água" (Arnaldo Antunes). Quando interrompia a música, o estudante respondia sobre uma questão relativa ao que foi trabalhado nos encontros anteriores e os outros complementavam, questionavam e discutiam.

\section{RESULTADOS E DISCUSSÃO}

No primeiro encontro, durante a atividade, poucos alunos e alunas mostraram algum conhecimento sobre o tema gravidez, nos aspectos físico-biológicos e social, sendo que os mais velhos sentiram-se mais confortáveis para fazer comentários. No entanto, no segundo encontro, ao final das apresentações dos cartazes, quando foi perguntado a eles a quem seria atribuída a responsabilidade do nascimento e cuidados com um bebê, no caso de uma gravidez na adolescência, poucos estudantes responderam que a responsabilidade da prevenção da gravidez seria da menina, a maioria atribuiu ao homem e à mulher. Isto mostra que, aparentemente, os adolescentes estão tendo uma percepção diferente dos homens e mesmo das mulheres do passado recente, quando atribuíam somente à mulher a responsabilidade contraceptiva e 
atendimento das necessidades da criança.

Entre os questionamentos feitos aos alunos no terceiro encontro, destacamos os referentes à cidadania e aspectos sócio-econômicos, solicitando que elencassem quais fatores são importantes para o desenvolvimento da criança/adolescente e as principais atividades para focar nesta fase da vida. A figura abaixo mostra, em porcentagem, os fatores elencados pelos alunos e alunas.

Chama a nossa atenção a diferença da proporção das meninas $(41,6 \%)$ em relação aos meninos $(8,7 \%)$ quanto à valorização da estrutura familiar, isto é, a importância que as meninas atribuem à presença dos pais para o bom desenvolvimento da criança. Entre os meninos, a preocupação com os recursos financeiros disponíveis para a construção de uma família se fez um fator prioritário (17,3\%). Ambos os sexos destacaram o acesso à educação alimentação e saúde como fatores básicos de sobrevivência, crescimento pessoal e direito à cidadania.

Neste sentido, verificamos uma sensibilização do público alvo, em nosso terceiro encontro quanto o papel da educação na formação de um indivíduo, tendo em vista que grande parte dos participantes elencou o estudo como uma das principais atividades para focar na infância/adolescência. Além disso, percebemos que a relação entre a educação e os fatores mínimos para o crescimento saudável descritos acima, estiveram intimamente relacionados à estrutura e renda familiar, o que corroboram com a visão de Dessen e Polonia (2007) de que a estrutura familiar e a escola desempenham papéis cruciais ao desenvolvimento humano, por permitirem ou inibirem o crescimento pessoal, cognitivo e ético dos indivíduos, o que se reflete diretamente sobre a estrutura e funcionamento das instituições sociais.

Cidadania em Ação: Revista de Extensão e Cultura, Florianópolis (SC), v. 2, n.2, jul./dez. 2018. 
Figura 1: Fatores abordados pelos participantes como essenciais para o desenvolvimento do indivíduo. Os tópicos apresentados foram apontados pelos participantes na etapa de avaliação dissertativa, sendo classificados por gênero e dispostos na figura por ordem de importância.

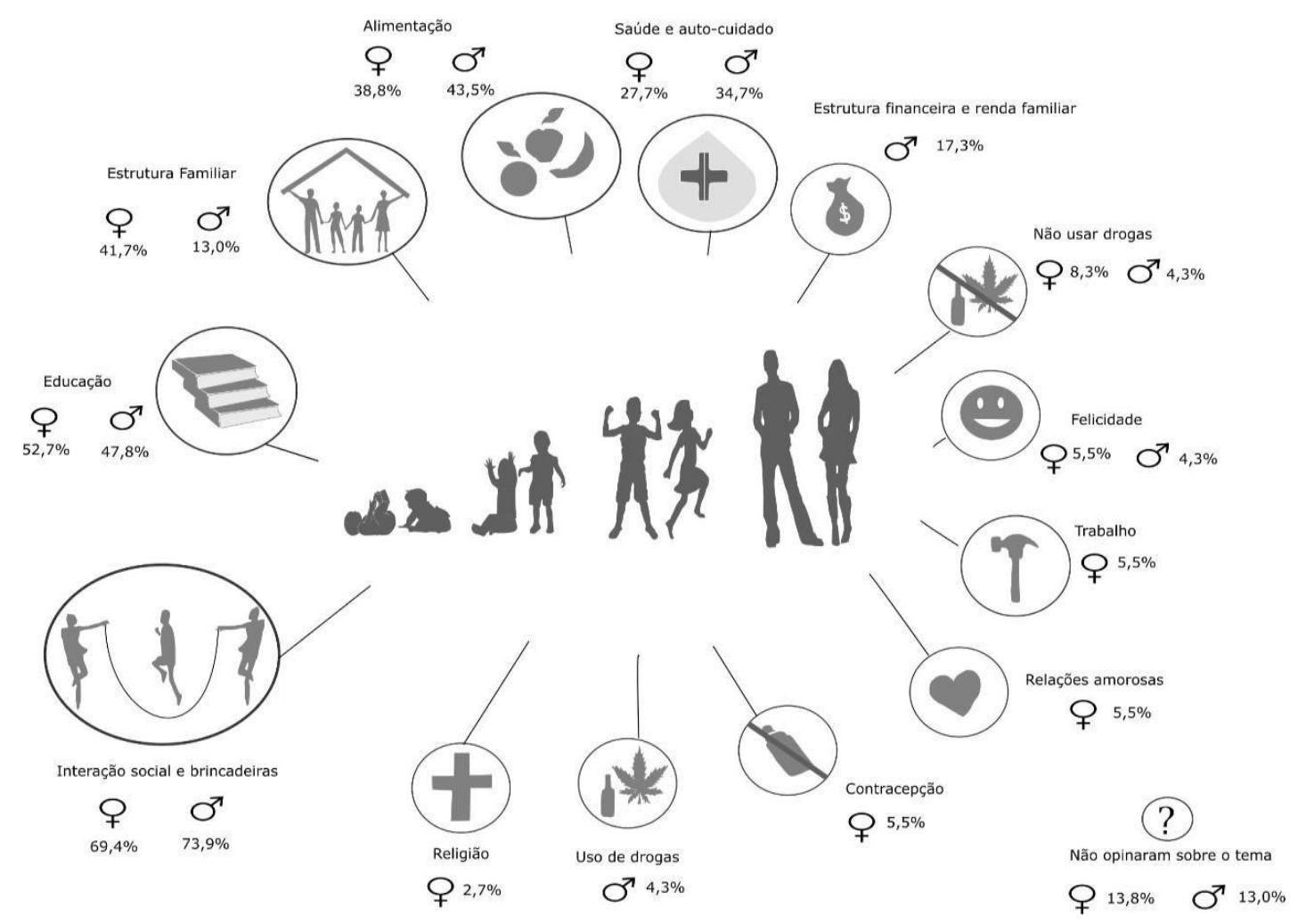

Também observamos que ambos os gêneros abordaram o lazer como principal atividade a ser vivida na infância/adolescência. $\mathrm{O}$ acesso brincadeiras durante o desenvolvimento vai além de um hobbie, preparando o indivíduo com regras para o convívio em sociedade e trabalho em equipe, bem como a ampliação dos processos cognitivos (PINTO et al., 2015). Nesta óptica, percebemos uma associação desses elementos, tendo em vista que 25\% das meninas e 17,3\% dos meninos enfatizaram a importância de brincadeiras coletivas e interação social com outras crianças/adolescentes. Além disso, a citação do trabalhar como fator importante para o desenvolvimento desponta para a análise de Bruner (1986) de que a brincadeira reflete a maneira como as crianças refletem sobre o mundo.

Adicionalmente, observamos uma possível associação da brincadeira com a prevenção de comportamentos abusivos, como o uso precoce de drogas, conforme relatado por Gomes et al, (1999), tendo em vista que em nossa observação o percentual de meninas/meninos que salientaram a importância da prevenção do uso de drogas lícitas-1́licitas foi maior do que o apontado pela parcela masculina que acreditava ser importante o uso das mesmas, sobretudo as

Cidadania em Ação: Revista de Extensão e Cultura, Florianópolis (SC), v. 2, n.2, jul./dez. 2018. 
de caráter ilícito. Isso é interessante, já que o bairro da escola, que também é o que a maior parte dos alunos residem, é considerado um hotspot para o tráfico.

Destacamos, em especial, uma resposta simples, dada por meninos e meninas, mas que pode dizer muito: que a criança e o adolescente precisam ser felizes. Segundo Giacomoni et al.(2014), crianças de escola pública responderam que ser feliz é ter satisfeitas as necessidades e desejos. Para Huebner e Gilman (2002), a satisfação infantil está na família, amizade, escola, ambiente e self, isto é, estar bem consigo mesmo. Levy e Jonathan (2010) sugerem que, para a criança, a família representa experiências positivas, como lazer.

As respostas dos alunos indicam que muitos que participaram das atividades estão refletindo sobre a importância de se planejar uma gravidez, já que apenas $13 \%$ das meninas e dos meninos não opinaram. Além disso, notamos que 5,5\% das meninas consideram a contracepção como importante nesta fase da vida. Também foi relatado pela professora das turmas que após o encerramento desta etapa do projeto, os/as estudantes continuaram discutindo sobre o tema e chegaram à conclusão da importância de estabelecer novas perspectivas além da maternidade/ paternidade precoce, considerando essa função uma alternativa de vida futura.

\section{CONSIDERAÇÕES FINAIS}

O despertar da consciência para a maternidade/paternidade precoce atrelado a percepção da valorização de outras atividades nesta etapa da vida sensibilizou os estudantes para o acesso a outras possibilidades do crescer, a exemplo dos estudos como uma ferramenta de desenvolvimento pessoal e social. Além disso, a proximidade geográfica da Universidade Estadual de Santa Cruz (UESC) com a escola em questão é um fator importante para a continuidade dessa abordagem, onde pretende-se trazer para perto uma realidade que para muitos ainda é distante. Assim, consideramos que os nossos objetivos estão sendo alcançados, pois já parece ter gerado um impacto positivo na vida dos adolescentes, considerando que estão em processo de transformações fisiológica e psicológica. Dessa maneira, outras práticas estão sendo planejadas para darmos continuidade ao projeto.

Cidadania em Ação: Revista de Extensão e Cultura, Florianópolis (SC), v. 2, n.2, jul./dez. 2018. 


\section{REFERÊNCIAS}

ALTMANN, H. Orientação sexual nos parâmetros curriculares nacionais. Revista Estudos Feministas, v. 9, n. 2, p. 576-585, 2001. Disponível em:

http://www.scielo.br/pdf/ref/v9n2/8641. Acesso 02 de Maio. 2018.

BRASIL. Ministério da Educação. Secretaria de Educação Básica. Base Nacional Comum Curricular - BNCC $1^{\mathrm{a}}$ versão. Brasília, DF, 2017. Disponível em: http://basenacionalcomum.mec.gov.br/. Acesso em: 19 fev. 2018.

BRUNER, J. Juego, pensamiento y lenguaje. Perspectivas, v.16, n. 1, p.79-86, 1986.

CARVACHO, I.E.; PINTO e SILVA, J.L.; MELLO, M.B. de. Conhecimento de adolescentes grávidas sobre anatomia e fisiologia da reprodução. Revista Associação Médica Brasileira, v. 53, n. 1, 2008.

DESSEN, M. A.; POLONIA, A. A família e a escola como contextos de desenvolvimento humano. Paidéia, V.17, n.36, 2007. Disponível em: http://www.scielo.br/pdf/paideia/v17n36/v17n36a03. Acesso em: 19 fev. 2018.

GIACOMONI, C.H.; SOUZA, L.K. de; HUTZ, C.S. A visão das crianças sobre a felicidade. Psicologia Escolar e Educacional, v. 18, n. 1, p. 143-150, 2014. Disponível em: http://www.scielo.br/pdf/pee/v18n1/v18n1a15. Acesso em: 25 fev. 2018.

GILMAN, R.; HUEBNER, E.S. Review of life satisfaction measures for adolescents. Behaviour Change, n.17, p. 178-195, 2000.

GOMES, R.; SILVA, C.M.F.P.D.; NJAINE, K. Prevenção à violência contra a criança e o adolescente sob a ótica da saúde: um estudo bibliográfico. Ciência \& Saúde Coletiva, v. 4, p. 171-181, 1999. Disponível em: https://www.scielosp.org/pdf/csc/1999.v4n1/171-181/pt. Acesso em: 25 fev. 2018.

HUEBNER, E. S.; GILMAN, R. An introduction to the multidimensional student's life satisfaction scale. Social Indicators Research, v. 60, p. 115-122. 2002.

LEVY, L.; JONATHAN, E.G. Minha família é legal? A família noimaginário infantil. Estudos de Psicologia, v. 27, n. 1, p. 49-56, 2010. Disponível em: http://www.scielo.br/pdf/estpsi/v27n1/v27n1a06. Acesso em: 25 fev. 2018.

ORGANIZAÇÕES UNIDAS NO BRASIL (ONUBR). Brasil tem sétima maior taxa de gravidez adolescente da América do Sul. 2017. Disponível em:

https://nacoesunidas.org/brasil-tem-setima-maior-taxa-de-gravidez-adolescente-da-americado-sul/. Acesso em: 19 fev. 2018.

PINTO e SILVA. Gravidez na adolescência: uma visão multidisciplinar, cap. 39 427-34. In: Saito MI, Silva LEV, Leal MM. Adolescência: prevenção e risco. 2 ed. São Paulo:

Atheneu; 2008. 
PINTO, A.C.S.; SCHENER, C.; SILVA, G.M.D.D.; SCHMITT, L.M.C. A importância do jogo na construção da cidadania. Unoesc \& Ciência-ACHS, p. 7-14, 2015. Disponível em: https://editora.unoesc.edu.br/index.php/achs/article/viewFile/8943/4891. Acesso em: 25 fev. 2018.

REBOUÇAS, J.S.S. Que ela cresça e apareça! - Gênero, corpo, poder e gravidez desejada entre as adolescentes do Colégio Estadual Rômulo Galvão, São Félix - BA (2012-2013). Trabalho de Conclusão de Curso. 2014. 105 f. (Curso Serviço Social) - Universidade Federal do Recôncavo da Bahia - Centro de Artes, Humanidades e Letras, Cachoeira, BA, 2014.

RIBEIRO, M. T. P.; SANTOS, M. B.; SANTOS, A. D. Características sociodemográficas e comportamentais relacionados à gravidez na adolescência no município de Jeremoabo, Bahia, Brasil. Scientia Plena, v. 11, n. 1, p. 1-12. 2015.

ROCHA, C.A. da Gravidez na adolescência e evasão escolar. Trabalho de Conclusão de Curso. 2009. 103 f. (Curso de Pedagogia) - Instituto de Biociências da Universidade Estadual Paulista "Júlio de Mesquita Filho" - Rio Claro, S.P, 2009.

RODRIGUES, R.M. Gravidez na Adolescência. Rev. Nascer e Crescer. v. 19, n. 3. p.1, 2010. Disponível em: http://www.scielo.mec.pt/pdf/nas/v19n3/v19n3a21. Acesso em: 25 fev. 2018.

ROMERO, K.T.; MEDEIROS, E.H.G.; VITALLE, M.S.D.S.; WEHBA, J. O conhecimento das adolescentes sobre questões relacionadas ao sexo. Rev. Assoc. Med. Bras, v.53, n.1, 2007. Disponível em: http://www.scielo.br/pdf/\%0D/ramb/v53n1/12. Acesso em: 25 fev. 2018.

SECRETARIA DA SAÚDE DO ESTADO DA BAHIA. Pesquisa realizada no Iperba mostra que gestantes adolescentes desconhecem a maioria dos métodos contraceptivos. 2016.

$<$ http://www.saude.ba.gov.br/novoportal/index.php?option=com_content\&view=article\&id=1 0642:pesquisa-realizada-no-iperba-mostra-que-gestantes-adolescentes desconhecem-amaioria-dos-metodos-contraceptivos\&catid=13: noticias\&Itemid=25>. Acesso em: 22 nov.

SILVA, V.C.; BARBIERI, M.; DE SOUZA APERIBENSE, P.G.G.; SANTOS, C.R.G.C. Gravidez na adolescência em unidades de saúde pública no Brasil: revisão integrativa da literatura. Adolescência e Saúde, v. 7, n. 4, 2010. Disponível em: http://www.adolescenciaesaude.com/detalhe_artigo.asp?id=247\&idioma=English\#. Acesso em: 25 fev. 2018. 